\title{
Landau-Like Damping in Rotating Pure Electron Plasmas
}

David A. Bachman and Roy W. Gould, Fellow, IEEE
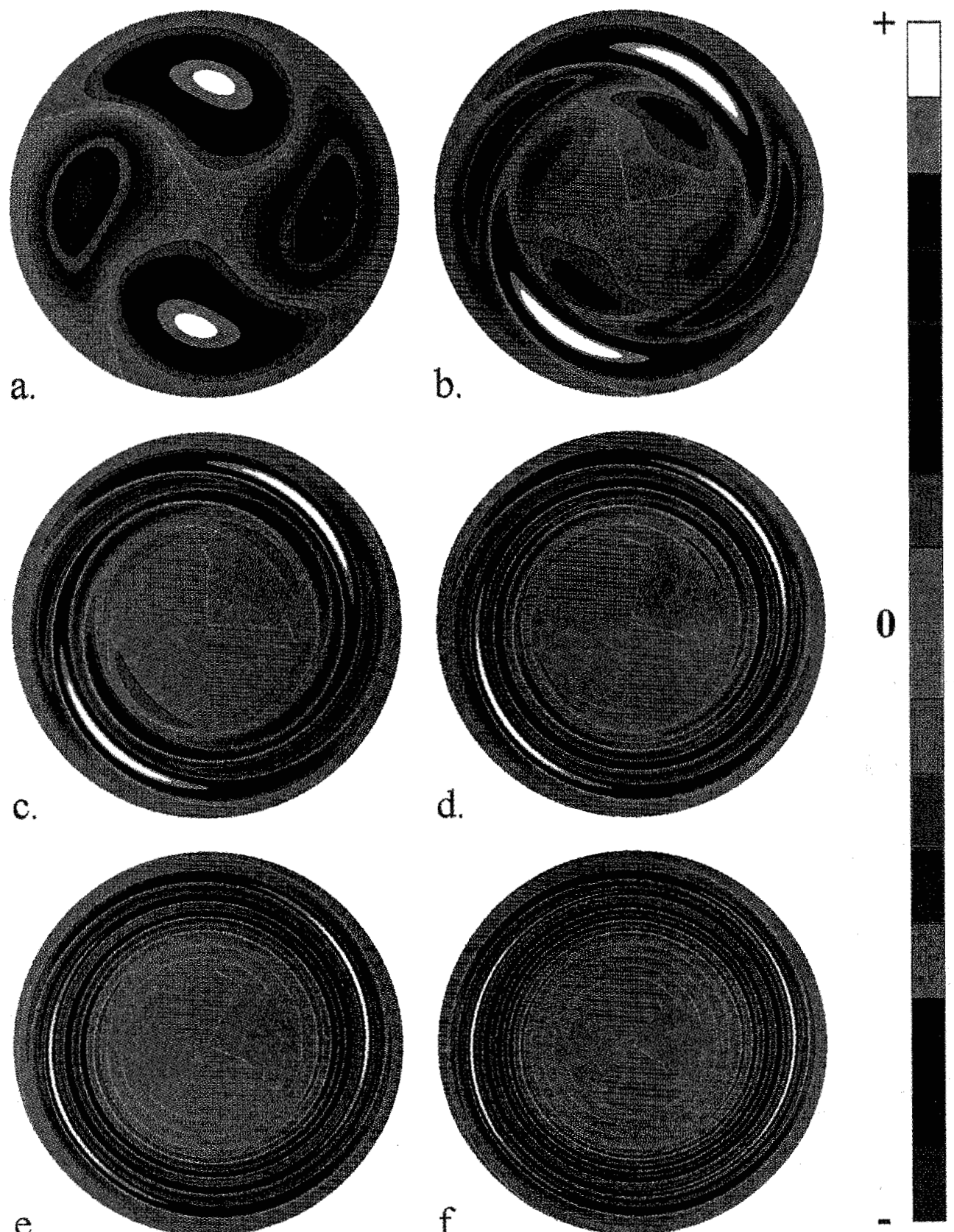

e.
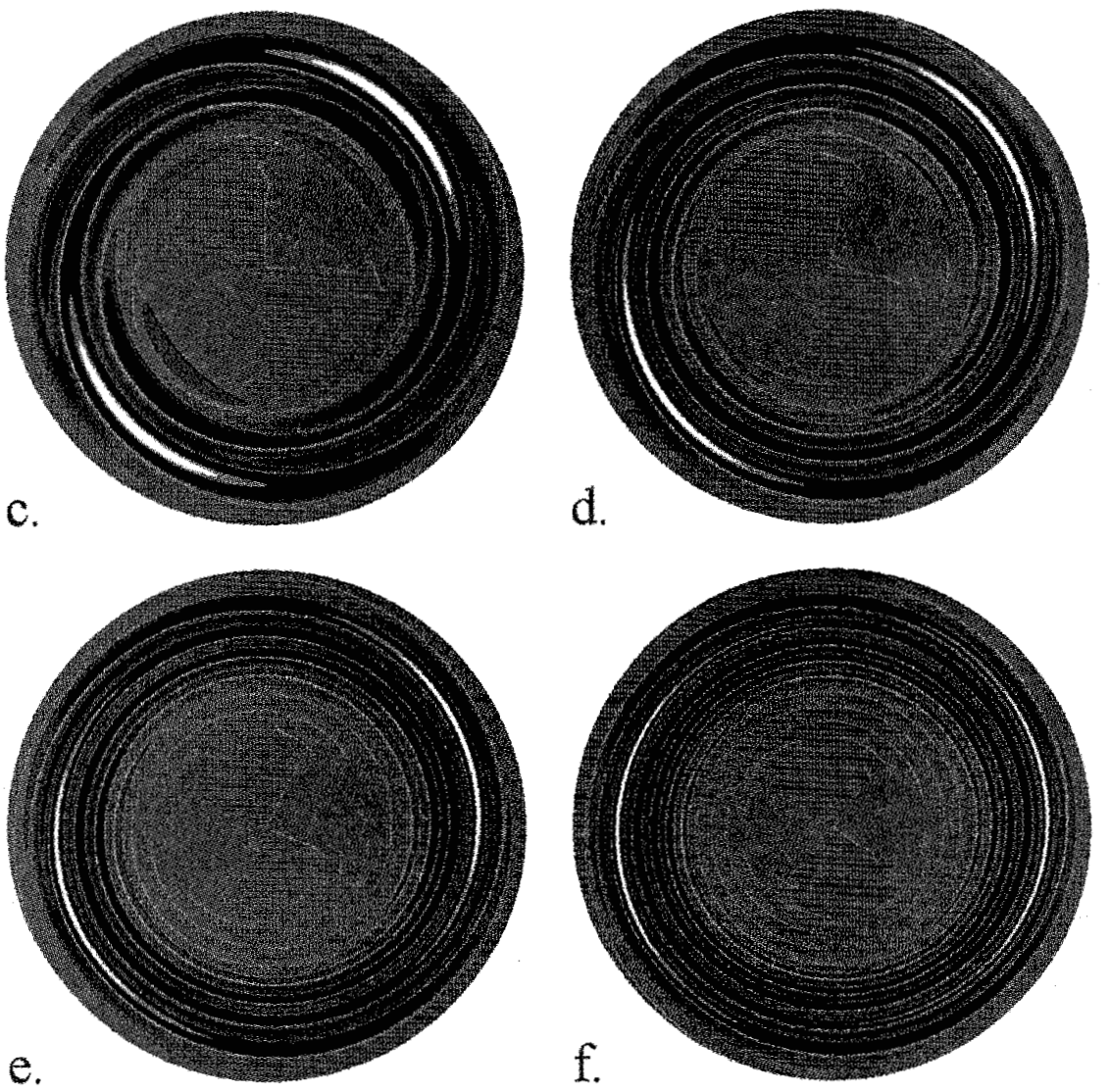

Fig. 1. Density perturbations at six successive times. The white color represents a high positive perturbation, while the black color represents an equal magnitude negative perturbation. Each successive color represents an equal change in perturbed density, with lines between colors representing constant perturbed density contours. Since the calculation is linear, the color scale is arbitrary. Between frames, the plasma rotates clockwise slightly more than half a revolution. 
Abstract-A numerical solution of the two-dimensional (2-D) linearized incompressible fluid equations has been used to study the response of a cylindrical pure electron plasma to an externally applied pulse. The plasma response to the pulse exhibits a nondissipative decay process. This decay is due to differential rotation of the plasma at different radii and is clearly shown by computer-generated displays of the perturbed density.

COLLISIONLESS damping of two-dimensional (2-D) disturbances in rotating fluids was predicted by Briggs et al. [1] and has recently been observed experimentally by Pillai and Gould [2]. This paper illustrates the physical mechanism responsible for collisionless damping in rotating fluids and nonneutral plasmas.

A cylindrical pure electron plasma is confined radially by a uniform magnetic field $B$ and axially with negatively charged trap electrodes [3], [4]. The electrostatic space charge selfrepulsion creates an outward radial force in the plasma which is balanced by an inward radial Lorentz force caused by steady-state rotation of the plasma within the uniform magnetic field.

In the drift approximation, three equations govern the dynamics of the plasma. These equations are the drift equation $\boldsymbol{v}=(-\nabla \phi \times \boldsymbol{B}) / B^{2}$, the continuity equation $(\partial n / \partial t)+$ $\boldsymbol{v} \cdot \nabla n=0$, and Poisson's equation $\nabla^{2} \phi=e n / \epsilon_{0}$, for the velocity $\boldsymbol{v}$, density $n$, and electric potential $\phi$, where $\epsilon_{0}$ is the permittivity of free space and $e$ is the magnitude of the electron charge. These equations are similar to the equations for 2-D incompressible inviscid fluid flow with the density analogous to the fluid vorticity $\varsigma=\nabla \times v$ and the electric potential analogous to the fluid stream function $\psi[1]$.

The steady-state angular velocity profile $\omega_{0}(r)$ is related to the assumed steady-state density profile $n_{0}(r)$ by $\omega_{0}(r)=$ $e /\left(\epsilon_{0} B_{0} r^{2}\right) \int_{0}^{r} n_{0}\left(r^{\prime}\right) r^{\prime} d r^{\prime}$. Assuming perturbations $\phi_{1}$ and $n_{1}$ with angular dependence $e^{i m \theta-i \omega t}$, the above equations may be linearized to obtain

$$
\begin{aligned}
& \frac{d^{2} \phi_{1}(r)}{d r^{2}}+\frac{1}{r} \frac{d \phi_{1}(r)}{d r}-\frac{m^{2}}{r^{2}} \phi_{1}(r)=\frac{e}{\epsilon_{0}} n_{1}(r) \\
& n_{1}(r)=\frac{m}{r B_{0}\left(m \omega_{0}(r)-\omega\right)}\left(\frac{d n_{0}(r)}{d r}\right) \phi_{1}(r) .
\end{aligned}
$$

Manuscript received July 14, 1995; revised October 3, 1995. This work was supported by the Office of Naval Research.

The authors are with the Department of Applied Physics, California Institute of Technology, Pasadena, CA 91125 USA.

Publisher Item Identifier S 0093-3813(96)02223-0.

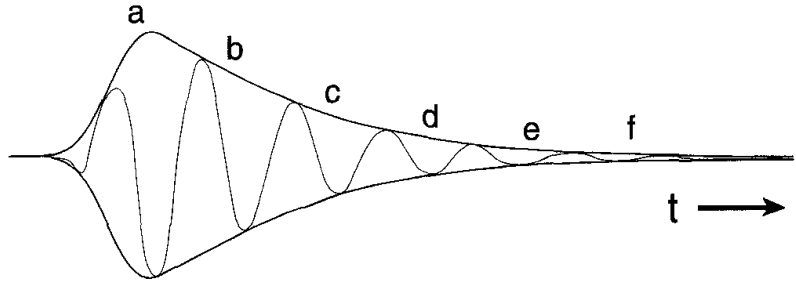

Fig. 2. Plot of the perturbed radial electric field at the wall in time. Each letter (a)-(f) corresponds to the time position of the frames in Fig. 1.

A short burst of voltage is applied with $m=2$ spatial symmetry to the electrodes which surround the plasma to initiate the perturbation at $t=0$. Solutions to (1) and (2) are obtained by numerically integrating along 500 steps in radius for 512 discrete complex frequencies $\omega$ that include a small fixed positive imaginary part used to make a discrete inverse Laplace transform.

Fig. 1 illustrates the perturbed density at six different times. A monotonically decreasing normalized density profile $n_{0}(r)=n_{0}(0)\left[1-3 r^{2}+3 r^{4}-r^{6}\right]$ has been assumed, which gives the steady-state angular velocity profile $\omega_{0}(r)=$ $\omega_{0}(0)\left[1-3 r^{2} / 2+r^{4}-r^{6} / 4\right]$, where $r$ is the plasma radius normalized to the wall radius. In this shear flow, the initial density perturbation is convected in the theta direction with different rates at different radii. As the radial layers shear, alternating bands of positive and negative density perturbations become thinner and more evenly distributed. The perturbed radial electric field at the wall, $E_{r}(t)$, shown in Fig. 2, is a weighted average of the density perturbations over the various radii. It decays in time due to phase mixing of the perturbed density $n_{1}(r, \theta, t)$ at different radial layers. This decay process is a spatial analogy to Landau damping in neutral plasmas [5].

\section{REFERENCES}

[1] R. J. Briggs, J. D. Daugherty, and R. H. Levy, "Role of Landau damping in crossed-field electron beams and inviscid shear flow," Phys. Fluids, vol. 13, no. 2, pp. 421-432, Feb. 1970.

[2] N. S. Pillai and R. W. Gould, "Damping and trapping in 2-D inviscid fiuids," Phys. Rev. Lett., vol. 73, no. 21, pp. 2849-2852, Nov. 1994.

[3] C. W. Roberson and C. F. Driscoll, AIP Conference Proceedings 175, Non-Neutral Plasma Physics. New York: Amer. Inst. Phys., 1988.

[4] J. Fajans and D. H. E. Dubin, AIP Conference Proceedings 331, NonNeutral Plasma Physics II. New York: Amer. Inst. Phys., 1994.

[5] L. Landau, "On the vibrations of the electronic plasma," J. Phys. (Moscow), vol. 10, no. 25, 1946. 